\section{Ausgezeichnet: Galenus-Preis 2015}

- Auch dieses Jahr wurde wieder der von Springer Medizin gestiftete Galenus-vonPergamon-Preis für pharmazeutische Innovationen verliehen. Die Preisträger wurden am 15. Oktober von Bundesgesundheitsminister Hermann Gröhe, dem Jury-Vorsitzenden Professor Erland Erdmann und seiner Vizepräsidentin Professor Marion Kiechle bei der "Springer Medizin Gala“ in Berlin geehrt. Mehr als 200 geladene Gäste erlebten im Axica am Brandenburger Tor hautnah mit, welche herausragenden Leistungen in der Arzneimittelforschung ausgezeichnet werden. Die Galenus-Preise wurden in vier Kategorien vergeben. Die 14-köpfige Jury entschied wenige Stunden vor der Verleihung über die Gewinner. Die Schirmherrschaft hatte Bundesforschungsministerin Johanna Wanka inne.

\section{Grundlagenforschung}

Den Preis in der Kategorie „Beste pharmakologische Grundlagenforschung" nahm in diesem Jahr Professor Jan Eric Siemens vom Pharmakologischen Institut der Universität Heidelberg entgegen. Mit seinem Team erforscht er, wie man Nervenzellen mithilfe von Botenstoffen weniger reizbar machen kann. Im Hirnzentrum wird der Schmerzrezeptor TRPV1 durch Entzündungsmediatoren, die bei Gewebeverletzungen oder pathologischen Veränderungen freigesetzt werden, sensibilisiert. Die Forscher entdeckten, dass man über die Aktivierung des GABA-B1-Rezeptors eine Sensibilisierung des TRPV1-Rezeptors verhindern oder aufheben kann.

\section{Primary Care}

In der Kategorie Primary Care ging der diesjährige Galenus-Preis an Nalmefen (Selincro ${ }^{\oplus}$ ) von Lundbeck. Hierbei handelt es sich um einen Opioidsystem-Modulator, mit dem sich der Alkoholkonsum bei abhängigen Erwachsenen vermindern lässt. Die Wirkung setzt rasch ein. Sowohl Gesamtalkoholkonsum als auch die Zahl der Tage starken Trinkens werden deutlich und dauerhaft reduziert.

\section{Specialist Care}

Als gezielte Therapie gegen Knochenmetastasen bei Prostatakrebs erhielt Radium223-dichlorid (Xofigo ${ }^{\circledast}$ ) von Bayer HealthCare den Preis im Bereich Specialist Care. Die Therapie bewirkt, dass Tumorzellen

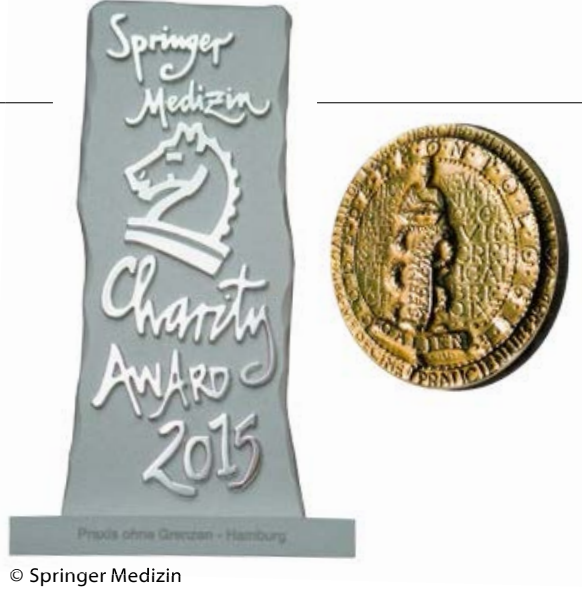

gezielt abgetötet werden, während das umgebende Gewebe und Knochenmark einer vergleichsweise geringen Strahlenbelastung ausgesetzt sind. Das Gesamtüberleben der Patienten und die Zeit bis zum Auftreten von symptomatischen skelettalen Ereignissen können auf diese Weise deutlich verlängert werden.

\section{Orphan Drugs}

Der Preis in dieser seit 2014 bestehenden Kategorie ging an Ataluren (Translarna ${ }^{\mathrm{TM}}$ ) von PTC Therapeutics. Dabei handelt es sich um die erste zugelassene Therapie mit kausaler Wirkung bei Duchenne-Muskeldystrophie mit zugrunde liegender Nonsense-Mutation. Das Medikament greift in die gestörte Bildung eines lebensnotwendigen Muskelproteins ein. Dadurch kann erstmals die Krankheitsprogression verzögert werden.

Uschi Kidane

\title{
CharityAward 2015 für karitatives Engagement
}

_ Seit 2009 zeichnet Springer Medizin ehrenamtliches Engagement in der Gesundheitsversorgung aus. Dieses Jahr gingen 49 Bewerbungen ein. Platz 1 ging an die "Praxis ohne Grenzen“ in Hamburg, in der 20 Ärzte mit anderen Ehrenamtlichen nicht versicherte Menschen medizinisch versorgen und sozial beraten. Platz 2 belegte das Projekt „Babylotse" der Stiftung des Katholischen Kinderkrankenhauses Wilhelmstift in Hamburg. Ehrenamtliche Mitarbeiter unterstützen bedürftige Familien während der Schwangerschaft und in der Phase nach der Krankenhausentlassung. Auf Platz 3 kamen die "Landarztmacher" aus der Region Arber, deren Mitglieder sich für den ärztlichen Nachwuchs engagieren, um dem Mangel in ländlichen Gebieten entgegenzuwirken.

Die Schirmherrschaft für den CharityAward hat seit Bestehen der Bundesgesundheitsminister inne, in diesem Jahr also wieder Hermann Gröhe.

Uschi Kidane.

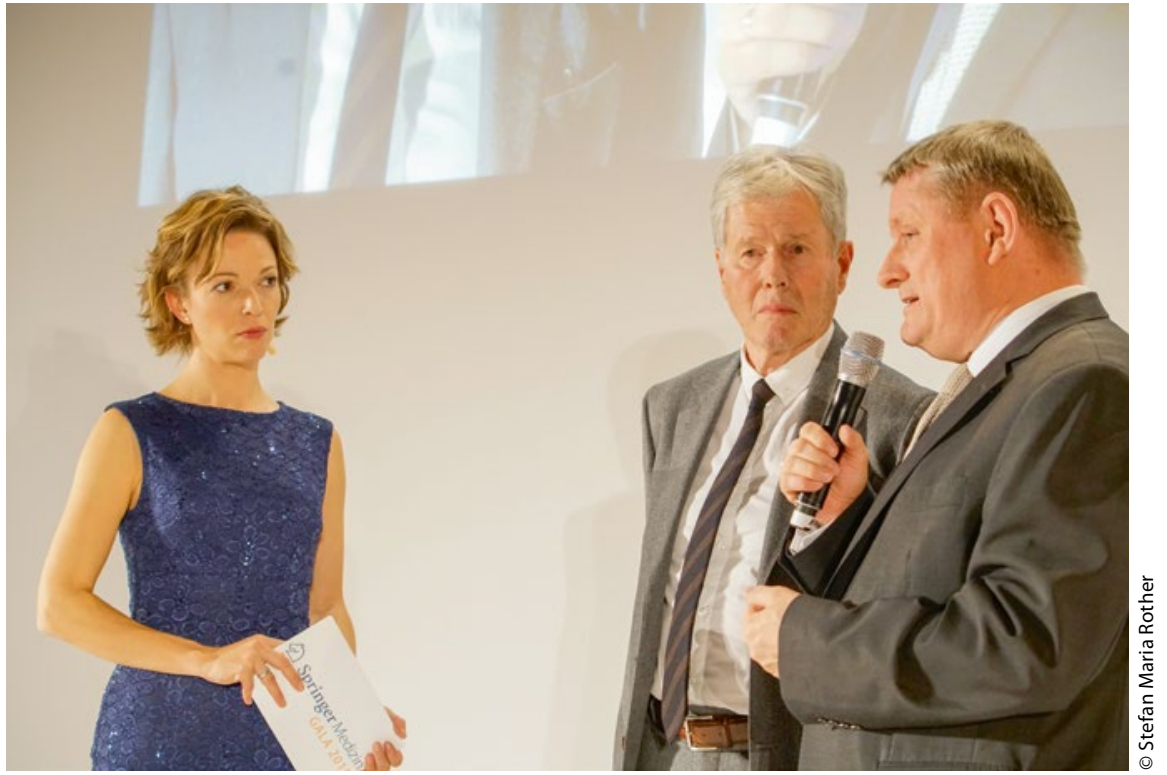

Professor Peter C. Ostendorf von „Praxis ohne Grenzen“ (Mitte) mit Moderatorin Anna Planken und Minister Hermann Gröhe. 